\title{
L'expédition antarctique Belge
}

In: Annales de Géographie. 1899, t. 8, n40. pp. 383-384.

Citer ce document / Cite this document :

Zimmermann Maurice. L'expédition antarctique Belge. In: Annales de Géographie. 1899, t. 8, n40. pp. 383-384.

doi : $10.3406 /$ geo.1899.6150

http://www.persee.fr/web/revues/home/prescript/article/geo_0003-4010_1899_num_8_40_6150 


\section{RÉGIONS POLAIRES}

L'expédition océanographique Allemande dans les mers Antarctiques. - L'expédition du I) Cucx est rentrée à Hambourg le 30 avril, après neuf mois de travaux couronnés du plus grand succès. Outre les résultats déja mentionnés ', il faul attirer l'attention sur les sondages opérés près de la terre d'Enderby, au delà du 64: deøré lat. S. $\left(64^{\circ} 13^{\prime}\right)$. Un dragage fournit une multitude de pierres provenant de la moraine de fond des glaciers antarctiques : ce sont les premiers faits qu'on ait recueillis sur la nature du continent antarctique. Les roches volcaniques font entièrement défaut; on ne recueillit que des fragments de granite, de gneiss, de schiste et de grès rouge ferrugineux. On ne possédait sur l'Océan Austral que 13 sondages au dela de $50^{\circ}$ S.; l'expédition du Valdivia en a ajouté 29 , dont 11 entre 3000 et $: 3: 30 \mathrm{~m}$. L'Océan du Sud, qu'on croyait être un bassin relativement bas, constitue un des plus profonds abimes océaniques, entre les 3 r $^{\circ}$ et $6 \mathbf{k}^{\prime}$ lat. $\mathrm{S}$. A 102 milles marins de la terre d'Enderby (position fixée à $63057^{\prime}$ ), on trouva encore $623 \mathrm{~m}$. Si la terre d'Enderby se trouve vraiment ì cette latitude, on aturait là une pente sous-marine d'une inclinaison qui ne se trouve fuère que dans les cas de terres volcanirques. Or les dépôts volcaniques manquent dans les dragages. Il y a donc peut-itre lieu de mettre en doute la position de la terre d'Enderby. Des observations de température eflectuées du 16 au 18 decembre, par $63^{\circ} \mathrm{S}$. et $3^{\circ} \mathrm{F}$. (ir., il ressort ce fait tres intéressant fu'immédiatement au-dessous des eaux slaceses de la surface (a partir de $130 \mathrm{~m}$.) jusqu'à plus de $2000 \mathrm{~m}$. de profondeur, règne une couche d'eau relativement chaude, de température variant entre $+0^{\circ}, \because \mathrm{C}$. et $+1^{\circ}, 6$. La partie inferieure des grands icebergs baigne donc, à ces haules latitudes, dans des eaux chaudes, et mème dans les régions antarcliques, la fusion en doit otre rapide. L'inattendu de ces conclusions, tant au point de vue des profondeurs que des températures, éveille naturellement le souvenir des observations de Nansen au pole opposé.

Le retour de l'expédition s'est fait par les îles Kerguélen, qu'aucun navire n'avait visitées depuis plusieurs années, et l'ile Saint-l'aul. Les sondages entre la terre d'Enderby et Kerguélen révélèrent des fonds extrêmement accidentés, où les profondeurs de $2360 \mathrm{~m}$. alternaient avec des abìmes de 3600 et de $: 3000 \mathrm{~m}$.

L'expédition antarctique Belge. - M. be Gerlache a envoyé à la Société Royale Belge de Géographice un comple rendu sommaire de son voyage 2. La Belgica n'avait pu quitter l'île des Etats que le 14 janvier 1898; dès le 22, en entrant dans le détroit de Bransfield, entre les South Shetlands et l'archipel de Dirk Gerrits\%, elle: perdit le matelot Wiencke, de Christiania, enlevé par une lame dans une tempête. La fin de l'été 1 ş४ ful consacrée à

1. Voir Ann. de Géog.. VII, 1898, chronirgue du 1; mars, p. 191 ; VIII, 1899, chronirge du 19 janvier, p. $9: 3$, du 13 mai, p. $28 \$$. La traduction du rapport officiel, paru dans le Deutsche Reicles-Anzeigerdu 1 ; mars 1898, se trouve dans Geog. Journ., XIII, juin 1899. P. 640-6;00.

2. Reprorluit par le Mouv. Gréog., $16^{\circ}$ année, 30 avril et 7 mai 1899 , et traduit dans le Geog. Journal, XIIl, juin 1899 , p. 6:30-634. 
l'exploration de l'archipel de Palmer, mal déterminé encore, et du détroit qui le sépare des terres de l'Est (terre de Graham). On opéra vingt débarquements dans tout ce complexe de terres, dont les coordonnées et les éléments magnétiques furent fixés. M. Racovrss lécouvit une faune antarctique entièrement nouvelle, représentée par un l’oduride, un Diptire et plusieurs minuscules Acariens.

S'avancant vers l'W., au delà de la terre d'Alexandre Ier, l'expédition tenta de gagner les hates latitudes; elle atteignit $71^{\circ} 31^{\prime} \mathrm{S}$. par $83^{\circ} 16^{\prime}$ long. W. Gr., mais le 10 mars elle se trouva bloquée, et dis lors, pendant ane année, elle dériva au gré des glaces. Cette dérive porta nettement vers l'W.; le navire se déplaça de 18 degrés dans cette direction, en se maintenant aux abords de $70^{\circ} 30^{\prime}$ lat. S.; la délivrance définitive n'eut lieu que le 14 mars 1899 .

Cette détention d'une année dans le pack antarctique assure une place d'honneur à l'expédition belge dans l'exploration des régrions polaires australes. Elle rapporte le premier ensemble dobservations d'hiver sur les glaces, les vents, le magnélisme, et à ce titre elle marque une date. Le pack, quoique le plus souvent trìs serré, 'resta toujours très mobile, au point que durant l'hiver toute excursion sur la glace parut impossible. Il semble que la houle, si forte dans les mer's australes, s'y fasse sentir beaucoup plus loin à l'intérieurde la banquise que dans les régions arctiques. Une seule pression fit courir des dangers au uavire, et pendant quelques minutes seulement. I.es tourmentes de neige et de vent étaient fréquentes : par vent du N., le temps restait presque toujours couvert, souvent brumeux, et assez doux; le froid venait par vent du S., avec le temps clair. Ces faits confirment ce que l'Antarctic et les expéditions antérieures nous avaient appris, et renforcent la probabilité de grandes masses de terres aux abords du pôle.

Les parages observés par la Belgicr durant sa dérive avaient été vus déjà par Biscoe, Bellingsmacsex, el le haleinier Walken; ils sont proches des points visités par Cook lors de sa pointe hardie jusqu'au $71^{\circ} 10^{\prime}\left(106^{\circ}: 4^{\prime}\right.$ long. W.; la Belyica ímergea des glaces par 103\%). L’apparence de terre signalée par Walkin (et non Wilkes) aux abords de $100^{\circ} \mathrm{W}$. et de $70^{\circ} \mathrm{S}$. ne se révéla par aucun indice : "Il est d'ailleurs à remarquer, écrit M. DE GenLAche, que notro dérive, presque aussi rapide vers le $S$. avec les vents du $X$. qu'clle l'était rers le $N$. avec les vents du $S$., ainsi que les sondages que nous avons effectués pendant notre dérive chaque fois que le temps a permis d'observer, contraignent a reculer de plusieurs degrés au S. les contours lyypothétiques du continent austral dans cette partie de la zone antarctique ". Cette conclusion, qui jette en même temps le doute sur les indices de terre induits de la relation de Cook, semble provisoirement le plus important résultat de l'expédition. Au point de vue sanitaire, il est i noter que pendant la nuit de l'hivernage, tous les membres de l'expédition sonfrirent de troubles cardiaques, et que le lieutenant Daxco, qui était atteint l'une maladie chronique du corur, succomba le: :j juin.

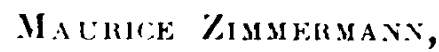
Arrécés dhistoire, et de grosgraphie.

Le Gerunt : Anmavd Colw. 